\title{
Questes
}

vestes Revue pluridisciplinaire d'études médiévales

$34 \mid 2016$

L'hiver

\section{Le solstice d'hiver et les traditions de Noël}

\section{Nadine Cretin}

\section{(2) OpenEdition}

\section{Journals}

Édition électronique

URL : http://journals.openedition.org/questes/4371

DOI : 10.4000/questes.4371

ISSN : 2109-9472

\section{Éditeur}

Les Amis de Questes

\section{Édition imprimée}

Date de publication : 15 décembre 2016

Pagination : 139-166

ISSN : 2102-7188

\section{Référence électronique}

Nadine Cretin, « Le solstice d'hiver et les traditions de Noël », Questes [En ligne], 34 | 2016, mis en ligne le 21 décembre 2016, consulté le 21 décembre 2020. URL : http://journals.openedition.org/questes/ 4371 ; DOI : https://doi.org/10.4000/questes.4371 


\section{Le solstice d'hiver et les traditions de Noël}

\section{Nadine CRETIN}

Historienne spécialisée en anthropologie religieuse

Nos fêtes hivernales trouvent en grande partie leur origine dans des célébrations ancestrales; de l'Antiquité à nos jours, elles ont été modifiées, détournées, abandonnées ou amplifiées. Déjà, dans l'ancienne Rome, le solstice d'hiver était célébré et donnait lieu aux Saturnales où l'on revivait l'Âge d'Or, temps mythique où tous les hommes étaient égaux. Ces fêtes, plus sages que ce que l'usage du mot laisse entendre, étaient marquées par de grands banquets domestiques où les esclaves, profitant des «libertés de décembre » comme l'écrivait le poète Horace ${ }^{1}$, mangeaient à la table de leur maître. Les Saturnales (qui duraient de un à plusieurs jours selon les époques, allant du 17 décembre au 23 dans sa plus grande extension), étaient connues à Rome depuis un temps immémorial. Dans une ambiance de paix, de partage et de fraternité ${ }^{2}$, c'était une période de trêve marquée par un temps d'arrêt dans les affaires tant publiques que privées où devaient cesser tout procès et même toute dispute individuelle ${ }^{3}$. À l'époque impériale, elles étaient suivies, une

\footnotetext{
${ }^{1}$ Horace, Satires, trad. François Richard, Paris, Garnier-Flammarion, 1967, livre II, 7, p. 202 : «Eh bien! nous sommes en décembre: profite, comme l'ont voulu nos ancêtres, de ta liberté ; parle », dit Horace à son esclave Dave.

${ }^{2}$ Cf. Macrobe, Les Saturnales, livre I, chapitre 1, trad. Charles Guittard, Paris, Les Belles Lettres, 1997, p. 5 ; Georges Dumézil, La Religion romaine archaïque, Paris, Payot, 1966 ; Charles Guittard, «Les Saturnales à Rome : de l'Âge d'Or au banquet de décembre », Pallas, «Banquets et représentations en Grèce et à Rome », n 61, 2003, p. 219-236.

${ }^{3}$ Valéry Raydon, Apologie du dieu Kronos. Du souverain prêtre au bienveillant boiteux, Paris, Le Labyrinthe, p. 75.
} 
dizaine de jours plus tard, par les Calendes de Janvier qui donnaient lieu à un grand repas la veille, à des échanges de vœux et d'étrennes, petits cadeaux et confiseries ${ }^{4}$, tout comme l'est devenu le Nouvel An. Elles engendraient de bruyantes mascarades extérieures où l'inversion était de mise : l'homme était déguisé en petite vieille ou en animal. Dans ces défilés, nous reconnaissons nos modernes carnavals, autres débuts d'année connus au Moyen Âge, qui allaient se caler à la veille du Carême et se multiplier dans les villes aux $\mathrm{XV}^{\mathrm{e}}-\mathrm{XVI}^{\mathrm{e}}$ siècles. Très tôt, les Pères de l'Église condamnèrent ces usages, tels, au IV siècle, saint Augustin pour qui l'usage du masque était une atteinte grave au créateur puisque l'homme avait été fait à la ressemblance de Dieu, Jean Chrysostome ou, plus tard, Césaire d'Arles au vi ${ }^{\mathrm{e}}$ siècle ${ }^{5}$. Mais ces traditions apparues vers 350 n'allaient pas disparaitre pour autant, comme nous allons le voir, et le Moyen Âge sera une période décisive dans l'histoire de cette transmission et de ces transformations. Le solstice était également marqué dans l'ancienne Rome par le culte au dieu Mithra venu de Perse, Sol Invictus («Soleil invaincu»). Ce dieu né de la pierre était justement fêté le 25 décembre : le culte fut reconnu religion officielle à Rome en 274 par l'empereur Aurélien. Quand, au IV ${ }^{\mathrm{e}}$ siècle, l'Église choisit de célébrer la Nativité de l'Enfant-Jésus, elle a placé l'Incarnation à cette même date du 25 décembre, entre autres à la faveur d’un rapprochement métaphorique du Christ, « lumière du monde » selon saint Jean l'Évangéliste (VIII, 12), qui reprenait la symbolique du soleil renaissant ${ }^{6}$. Précédemment, l'Église ne s'était attachée qu'à la Résurrection. Saint Luc, qui rapporte dans son évangile la mangeoire de Bethléem et l'annonce aux bergers (II, 1-20), ne

\footnotetext{
${ }^{4}$ Ovide, Fastes, Livre 1, v. 185 sq.

${ }^{5}$ Daniel Fabre, Carnaval ou la fête à l'envers, Paris, Gallimard, 1992, p. 27-31.

${ }^{6}$ La première attestation figure en 354 dans un chronographe établi à Rome vers 336, le calendrier «philocalien »d'après le nom de Furius Dionysius Philocalus, l'artiste grec qui l'illustra pour un aristocrate chrétien nommé Valentin..
} 
donne pas de date, pas plus que saint Matthieu qui, lui, parle de la visite des Mages (II, 1-12) ${ }^{7}$.

$\mathrm{Au}$ moment de l'institution de la fête dans les années 330, l'assemblée chrétienne était conviée à une messe du jour de Noël que le pape célébrait à Rome en la basilique Saint-Pierre, ainsi nommée en souvenir du premier chef de l'Église ${ }^{8}$. La messe de la nuit apparut en 440 à l'église Sainte-Marie-Majeure près de laquelle fut édifié un oratoire qui contenait une réplique de la crèche de Bethléem : Sixte III célébra là la messe ad galli cantum, au chant du coq ${ }^{9}$. Un troisième office, à l'aurore, était célébré par le pape à l'église Sainte-Anastasie, au cœur du quartier byzantin, en l'honneur d'Anastasie, martyre de Dioclétien à Sirmium (Dalmatie), dont c'était la fête le 25 décembre. C'est là, déjà attestée par le pape Grégoire le Grand (†604), l'origine des trois messes de Noël qui auront la vie longue ${ }^{10}$. Ainsi, garda-t-on jusqu'au début du $\mathrm{xx}^{\mathrm{e}}$ siècle l'habitude d'assister à plusieurs messes d'affilée. Pour la période qui nous intéresse, citons l'exemple de Montfaucon au baillage de Vitry en Normandie, où, en 1395, un paroissien fut condamné pour être sorti dans

\footnotetext{
${ }^{7}$ On détermina la naissance du Christ d'après l'an 15 de Tibère (28-29) qui coïncidait avec sa trentième année, selon l'Evangile de saint Luc (III, 1 et III, 23). Au VIe siècle, le moine romain d'origine scythe Denys le Petit ( $\dagger$ entre 526 et 556) fixa l'an I de notre ère à la naissance du Christ, 753 ans après la Fondation de Rome. Mais la naissance du Christ aurait eu lieu quelques années plus tôt. La diffusion de l'ère chrétienne, quant à elle, ne doit rien au legs romain, mais à l'usage qu'en firent les réformateurs insulaires qui l'importèrent en Angleterre. De là, l'usage passa en GauleGermanie de Pépin de Herstal au VIII ${ }^{\mathrm{e}}$ siècle, et se diffusa aux IX ${ }^{\mathrm{e}}-\mathrm{X}^{\mathrm{e}}$ siècles grâce au corps épiscopal avant de se répandre dans les actes "privés »: (Jean-Patrice Boudet, Olivier Guyot-Jeannin, « Temps romain, temps chrétien, temps de l'État », Rome et l'État moderne européen, École Française de Rome, 2007, p. 77.

${ }^{8} \mathrm{Ce}$ sanctuaire, élevé par Constantin pour vénérer le premier évêque de Rome au Vatican sur le lieu présumé de son martyre, et consacré en 326 par le pape Sylvestre, devint très tôt un lieu de pèlerinage. Pierre Grimal, Voyage à Rome, promenades romaines, Paris, Robert Laffont, 2004, p. 635-636. La basilique que l'on connaît aujourd'hui fut édifiée au XVI siècle, sous le pape Jules II.

${ }^{9}$ Robert Féry, Jours de Fêtes, Paris, Seuil, 2008, p. 32.

${ }^{10}$ Pierre Jounel, L'Église en prière, vol. IV, « La liturgie et le temps », Paris, Desclée, 1983 , p. 97.
} 
la nuit de Noël avec d'autres afin d'aller se chauffer près du cimetière «avant la deuxième messe ${ }^{11} »$.

En explorant les différents thèmes rendant chacun compte de la transformation importante des fêtes hivernales antiques au Moyen Âge, nous étudierons d'abord la fête de Noël, puis les jeux et représentations qu'elle occasionnait, ses traditions sociales et domestiques et nous finirons par les « Douze Jours ».

\section{«Al Naël Deu », à la naissance de Dieu}

L'une des premières attestations du mot qui donna « Noël » figure dans le Voyage de saint Brendan au tout début du XII siècle : al Nael Deu, à la Naissance de Dieu. Le mot vient du latin Natalis. Un peu plus tard au XIII ${ }^{\mathrm{e}}$ siècle (vers 1262), Rutebeuf, dans la Vie de Sainte Marie l'Egyptienne, écrit du Christ : C'est cil qui nasquit a Noei. L'orthographe définitive du mot Noël, avec le tréma qui note la diérèse, n'a été fixée qu'en 1762 dans la quatrième édition du Dictionnaire de l'Académie française $^{12}$. Avant l'apparition du mot, on parlait simplement de la fête de la Nativité du Seigneur (Natalis Domini). Celle-ci était très aimée comme le prouvent les nombreuses représentations des églises ou musées qui nous sont parvenues (tableaux, sculptures, vitraux). On trouve également plusieurs sculptures des porches et vitraux de la cathédrale de Chartres du XIII ${ }^{\mathrm{e}}$ siècle, «apogée de la chrétienté » selon Jacques Le Goff ${ }^{13}$, ou, plus tard, le livre d'heures de Béatrice de Rieux conservé à la bibliothèque des Champs libres de Rennes (ms. 2044, vers 1390), ou le tableau La Nativité de Robert Campin, dit le maître de Flémalle (vers 1435), au musée des

\footnotetext{
${ }^{11}$ Roger Vaultier, Le Folklore pendant la Guerre de Cent Ans d'après les lettres de Rémission du Trésor des Chartes, Paris, Guénégaud, 1965, p. 85.

${ }^{12}$ Tome II, p. 213.

${ }^{13}$ Jacques Le Goff, Le XIII siècle, l'apogée de la chrétienté (v. 1180-v. 1330), Paris, Bordas, 1982.
} 
Beaux-Arts de Dijon ${ }^{14}$. Noël! était par ailleurs un cri de réjouissance connu au Moyen Âge vers $1300^{15}$. Il pourrait venir de novella, «(bonne) nouvelle». Certains font dériver le nom dialectal Noué, Noé de « nouveau », « renouveau ».

La période de Noël, appelée le plus souvent «Douze Jours» (de Noël à l'Épiphanie), portait différents noms suivant les régions. Cette période «calendale » (de calendas, début du mois), qualificatif toujours en usage en Provence pour la situation de l'époque dans le calendrier à l'aube du Nouvel An, s'appelait Chalende en Dauphiné, Calendo en Provence, mais son nom était fréquemment dérivé de natalis : Nadal en Languedoc, Nau dans l'Ouest de la France (Charente, Poitou, Anjou), Nedeleg en Bretagne ${ }^{16}$.

Les représentations de la naissance de l'Enfant-Jésus et de ses adorateurs apparurent à partir $\mathrm{du} \mathrm{IV}^{\mathrm{e}}$ siècle sur des sarcophages à Rome, Milan et Arles, toujours visibles dans des musées lapidaires ou des églises, et se multiplièrent dans les enluminures des livres liturgiques. L'une des plus anciennes figurations de la Nativité, avec l'âne et le bœuf ainsi que l'adoration des bergers au clair de lune, est un fragment de sarcophage romain de la fin du $\mathrm{IV}^{\mathrm{e}}$ siècle, conservé à Arles, au musée lapidaire ${ }^{17}$.

Le Moyen Âge allait s'emparer avec cœur de l'aspect rural de la Nativité, si parlant. Déjà, saint Ambroise, évêque de Milan de 374 à 397, avait choisi le thème dans son Traité sur l'évangile de saint Luc:

Qu'il soit dans les langes, vous le voyez; vous

\footnotetext{
${ }^{14}$ Huile sur bois, $86 \times 72 \mathrm{~cm}$.

${ }^{15}$ Guillaume de La Villeneuve, «Les Crieries de Paris », 109, dans Fabliaux et contes des poètes françois des XI ${ }^{e}, X^{e}{ }^{e}, X I I I^{e}, X I V^{e}$ et XV $V^{e}$ siècles, éd. Étienne Barbazan, Paris, B. Warée oncle, 1808, vol. II, 282 : «Noël! Noël! à moult granz cris».

${ }^{16}$ Arnold Van Gennep, Le Folklore français, Cycle des Douze Jours de Noël aux Rois, Robert Laffont, «Bouquins », 1999 [Picard, 1958], p. 2314 et suiv., selon de minutieuses enquêtes de terrain.

${ }^{17}$ Françoise Lautman, Crèches et traditions de Noël, Édition de la RMN, Paris, 1986, p. 25.
} 
ne voyez pas qu'il est dans les cieux. Vous entendez les vagissements de l'enfant, vous n'entendez pas les mugissements du bœuf qui reconnaît son propriétaire et l'âne la crèche de son Maître ${ }^{18}$.

L'âne et le bœuf furent associés définitivement à la crèche, comme le montre la description d'un texte apocryphe, l'évangile du Pseudo-Matthieu (premier quart du VII siècle) dit «l'évangile de l'enfance », au chapitre XIV :

Marie [...] déposa l'enfant dans une crèche et le bœuf et l'âne, fléchissant les genoux, adorèrent celui-ci. Alors furent accomplies les paroles du prophète Isaïe disant: Le bouf a connu son propriétaire, et l'âne le crèche de son maître, et ces animaux, tout en l'entourant, l'adoraient sans cesse ${ }^{19}$.

De même que, selon l'évangile de Luc, les bergers furent les premiers avertis par les anges la nuit-même de la Nativité alors qu'ils dormaient dans les champs, les deux animaux domestiques ont contribué à la réputation de la scène de la Nativité. Tout dans cette naissance divine baigne dans la simplicité, à l'image des bergers qui étaient des hommes très modestes et proches du peuple.

Un autre facteur exotique aida à la figuration de la scène de la Nativité : il s'agit de la visite des Mages, qui étaient venus d'Orient en suivant l'étoile selon saint Matthieu. Si leur nombre a pu varier au début, on les fixa progressivement à trois en s'alignant sur les trois cadeaux cités

\footnotetext{
${ }^{18}$ Saint Ambroise, Traité sur l'évangile de saint Luc, livre II, 42, Paris, Le Cerf, 1956, p. 92.

${ }^{19}$ Écrits apocryphes chrétiens, éd. François Boyon, Pierre Geoltrain et Sever J. Voicu, Paris, Gallimard, 1997, p. 134. Nous soulignons. En réalité, la prophétie d'Isaïe n'avait rien à voir avec la Nativité : "Le bœuf connaît son possesseur, et l'âne la crèche de son maitre, [...] mon peuple ne comprend pas » (I, 3). Le Pseudo-Matthieu mentionne également une prophétie d'Habacuq (III, 2) : «Tu te manifesteras au milieu de deux animaux ». Mais cette traduction n'existe que dans le texte grec de la Septante, alors que le sens du texte hébreu est différent : «En notre temps (au milieu des années), fais connaître ton œuvre ».
} 
dans l'Évangile : l'or, l'encens et la myrrhe. Sur le sarcophage d'Arles en marbre blanc cité plus haut la scène supérieure représente en son centre l'Enfant dans un berceau, la Vierge assise près de là sur la gauche, l'âne et le bœuf derrière le berceau et sur la droite, debout, un berger à demi-vêtu reconnaissable à sa houlette. Au niveau inférieur, les trois mages sont habillés comme des Perses : justaucorps à volants et bonnets phrygiens ${ }^{20}$. L'un montre l'étoile. Dans les premiers siècles, ils étaient fréquemment représentés ainsi, par exemple sur une mosaïque du $\mathrm{VI}^{\mathrm{e}}$ siècle de l'église Saint-Apollinaire-le-Neuf à Ravenne. Par la suite, de nombreuses légendes amplifièrent les richesses de ces personnages exotiques comme le laissaient supposer leurs précieux cadeaux. Comme dans les Collectanea attribuées à Bède le Vénérable, on leur donna des noms d'origine perse-Melchior, Gaspar et Balthazar-et une apparence les faisant appartenir aux trois continents alors connus (l'Europe, l'Afrique et l'Asie) et aux trois âges de la vie, On en fit très tôt des rois en respectant, à la suite de Tertullien (160-220) et du le psaume 72 de l'Ancien Testament - «Les rois de Tarsis et des îles rendront tribut. Les rois de Saba et de Seba feront offrande... »-, ainsi que la prophétie d'Isaïe (LX, $3)$ - «Les rois se prosterneront sur ta clarté naissante ${ }^{21} »$. Leur célébrité, accrue dès lors, ne faillit pas, même si ce ne fut qu'à partir du $\mathrm{X}^{\mathrm{e}}$ siècle qu'on les représenta avec des attributs royaux, et leurs reliques allaient être vénérées comme celles de véritables saints ${ }^{22}$. Dans sa Chronique, qui

\footnotetext{
${ }^{20}$ Henri Leclercq, Dictionnaire d'Archéologie chrétienne et de Liturgie, Letouzey et Ané, 1931, « Mages », vol. 10-1, col. 980-1067.

${ }^{21}$ Richard C. Trexler, Le Voyage des mages à travers l'histoire, pref. de Jacques Le Goff, Paris, Armand Colin, 2009, p. 25 et suiv.

${ }^{22}$ Robert Féry, Jours de Fêtes, op. cit., p. 35 ; Mathieu Beaud, «Les Rois mages. Iconographie et art monumental dans l'espace féodal $\left(\mathrm{X}^{\mathrm{e}}-\mathrm{XII}^{\mathrm{e}}\right.$ siècle) $»$, Bulletin $d u$ centre d'études médiévales d'Auxerre, vol. 17, $\mathrm{n}^{\circ} 1$, 2013. URL: http://cem.revues.org/13079. Voir aussi la communication de cet auteur «Les Mages à l'époque romane: entre imaginaire et dogme », dans Les Rois mages, dir. JeanMarcVercruysse, Actes du colloque des 25 et 26 mars 2010, Arras, Artois Presses Université, 2011, p. 59-74.
} 
connut un grand succès en Normandie et en Angleterre, Robert de Thorigny, devenu abbé du Mont-Saint-Michel en 1154, écrivit :

En l'année 1158, on découvrit dans une antique chapelle, près de la ville de Milan, les corps des trois Mages [...] et par crainte de Frédéric [Barberousse], empereur d'Allemagne, qui se disposait à assiéger Milan, on les releva et on les déposa dans cette ville. En 1164, Renaud de Dassel, archevêque élu de Cologne et chancelier de Frédéric, [...] transféra les corps de Milan à Cologne. Ces corps qui avaient été embaumés étaient conservés intacts [...] Saint Eustorge (315-331), qui les avait reçus d'un empereur, les avait transportés de Constantinople à Milan avec une table sur laquelle ils étaient étendus, dans un petit chariot que tiraient deux vaches ${ }^{23}$.

Le transfert de leurs reliques à Cologne en 1164 répondait au souhait de l'empereur Frédéric Barberousse après le sac de la ville de Milan en 1162, et la dimension royale des personnages était évidemment de grande importance.

\section{Jeux et représentations}

Grâce aux représentations hautes en couleur que le thème engendrait, les mises en scène de la naissance de l'Enfant-Jésus se développèrent au cours des célébrations médiévales de Noël et de l'Épiphanie. Elles naquirent des antiennes dialoguées, tel le tropaire de l'abbaye Saint-Martial de Limoges, manuscrit du XI siècle où se trouve la plus ancienne forme connue d'une Nativité jouée ${ }^{24}$. Ces jeux, dits liturgiques, sont introduits au cours des cérémonies de Pâques ou de Noël pour les rendre plus accessibles aux fidèles et plus animés. Encouragé par

${ }^{23}$ Chronique de Robert de Thorigny, citée par Joseph Daoust, Encyclopédie du Catholicisme, Hier, Aujourd'hui, Demain, Encyclopédie publiée sous la direction du Centre interdisciplinaire des Facultés catholiques de Lille, t. VIII, 1979, col. 140.

${ }^{24}$ Troparium-prosarium ad usum Sancti Martialis Lemovicensis, Paris, BnF, ms. 887, Françoise Lautman, op. cit., 1986, p. 37. 
le bilinguisme (latin/langue vulgaire), le jeu dialogué, donné dans les églises dès la fin du $\mathrm{X}^{\mathrm{e}}$ siècle dans toute l'Europe (appelé officium ou ceremonia mais jamais «drame liturgique »), contribuait à la splendeur d'une liturgie déjà florissante avec les nouvelles compositions poétiques qu'étaient les tropes ou séquences ${ }^{25}$. Ces jeux se sont particulièrement développés à la fin du $\mathrm{XI}^{\mathrm{e}}$ et au XII $\mathrm{XI}^{\mathrm{e}}$ siècle. Le thème de l'adoration des Mages et leurs cortèges de plus en plus pompeux augmentèrent la popularité de la représentation de la Nativité qui devenait très visuelle. Ainsi, un manuscrit de la bibliothèque d'Orléans datant du XIII siècle, qui appartenait à l'abbaye de Fleury et dont certaines parties remontent au moins au XII (vers 1150), comporte dix drames liturgiques avec airs notés sur portée, dont une célèbre Adoration des Mages ${ }^{26}$. En outre, comme le constate Françoise Lautman, ethnologue spécialisée dans les faits religieux ${ }^{27}$, La Légende dorée (fin du XIII ${ }^{\mathrm{e}}$ siècle) du dominicain Jacques de Voragine a contribué à la diffusion dans la culture médiévale de « tout le légendaire anecdotique autour de la naissance et de l'enfance du Christ, qui a sa source dans les Évangiles apocryphes et qui relève du même souci narratif concernant les aspects familiers de ces événements ${ }^{28} »$. Ces jeux conduiront progressivement à la représentation de la crèche. L'exemple de la messe de Noël au Greccio avec saint François d'Assise en 1223, est bien connu. «Afin d'augmenter la dévotion des habitants ${ }^{29}$ » comme l'a

\footnotetext{
${ }^{25}$ Marie-Noël Colette, «Les jeux liturgiques. Sens et représentations », Revue de Musicologie, vol. 8611, 2000, p. 6.

${ }^{26}$ Recueil d'Hymnes et de Drames liturgiques de l'abbaye de Fleury-sur-Loire, ms. 10, fol. 205-214. Fleury est devenu Saint-Benoît-sur-Loire au cours du VIII siècle, à la suite du transfert des reliques du saint italien en 660 sous la conduite du moine Aigulfe.

27 Directeur de recherche honoraire, GSRL-CNRS; Commissaire de l'exposition «Crèches et traditions de Noël » au Musée National des Arts et Traditions populaires (21 octobre 1986-16 février 1987).

${ }^{28}$ Crèches et traditions de Noël, Paris, RMN, 1986, p. 36 et 39.

${ }^{29}$ Saint Bonaventure, Vie de saint François, (texte approuvé en 1263), trad. Damien Vorreux, Paris, Éditions Franciscaines, 1951, p. 178.
} 
écrit son biographe saint Bonaventure vers 1263et après avoir obtenu l'autorisation du pape (Honorius III), le diacre François choisit d'exporter pour la première fois la messe de la Nativité hors d'une église, dans une grotte des Abruzzes, en présence d'un âne et d'un bœuf. Une mangeoire servait d'autel et la foule se pressait «à la clarté des torches étincelantes et au son des cantiques résonnant haut et clair». Le clerc Thomas de Celano $(\dagger 1265)$, sur qui saint Bonaventure s'est appuyé, précise même que la forêt retentissait des voix et que les roches répondaient aux cris de jubilation $^{30}$. Celano prend soin de mentionner que le foin de la crèche, conservé par les fidèles, était ensuite utilisé comme remède pour soigner les accouchées et pour guérir les animaux malades. Cet ajout prouve la part importante de la «superstition » liée aux croyances dans la religion médiévale : sous les apparences du culte chrétien, les pratiques ancestrales persistaient, malgré les nombreuses tentatives de l'Église pour les éradiquer. Il est remarquable ici que ce soit un clerc qui mentionne ces pratiques sans porter de jugement négatif. ${ }^{31}$.

Avec une grande liberté, l'offrande des bergers s'intégra parfaitement à la liturgie des messes de minuit. L'origine des cadeaux des bergers, qui ne figurent pas dans l'Évangile contrairement à ceux des Mages, découle des mystères joués sur les parvis de la fin du XIV ${ }^{\mathrm{e}}$ au XVI ${ }^{e}$ siècle, comme celui de la Passion d'Eustache Marcadé (dit aussi Mercadé) ou celui d'Arnoul Gréban, originaire du Mans, joué à Paris

\footnotetext{
${ }^{30}$ Thomas de Celano, Vie de François, éd. Jacques Dalarun, François d'Assise. Écrits, Vies, témoignages, Paris, Cerf, 2010, p. 571 ; Charles M. de La Roncière, «La Nativité dans la dévotion de saint François d'Assise » La Nativité et le temps de Noël, Antiquité et Moyen Âge, dir. Gilles Dorival et Jean-Paul Boyer, Aix-en-Provence, Presses universitaires de Provence, 2003, p. 231-243.

31 Comme Jean-Claude Schmitt, nous mettons ici des guillemets au mot «superstition », phénomène que l'esprit rationaliste d'aujourd'hui raille volontiers, mais que le Moyen Âge considérait comme essentiel pour contrôler les aléas de l'avenir. («Les superstitions », Histoire de la France religieuse, Jacques Le Goff et René Rémond, vol. 1, Paris, Seuil, 1988, p. 417-453.
} 
avant $1452^{32}$. Ce mystère fut repris dans le Grant Kalendrier et compost des bergiers en 1491 réédité à de nombreuses reprises. Les mystères étaient des drames profanes et réalistes qui se voulaient respectueux des sources religieuses, mais leurs éléments comiques les maintenaient hors des sanctuaires. En raison du climat, il y avait moins de mystères joués au moment de Noël qu'à Pâques, à la Pentecôte ou l'été ${ }^{33}$. Après l'Introït ou avant l'offertoire, les jeux liturgiques commençaient souvent avec la question Quem vidistis, pastores, dicite? (Qui avez-vous vu, bergers, dites ?), établie sur le modèle de Pâques Quem quaeritis in sepulchro? (Qui cherchez-vous dans la tombe ?). Cette formule n'a son origine ni dans la Vulgate, ni dans les évangiles ${ }^{34}$. Dans les drames liturgiques, c'est le jeu, la performance qui introduit le rire au sein du plus sacré : le personnage de l'Enfant-Jésus ${ }^{35}$. De tels jeux, nombreux dans la plupart des régions jusqu'au XVIII ${ }^{\mathrm{e}}$ et même $\mathrm{XIX}^{\mathrm{e}}$ siècle - comme à Carcassonne en 1839 selon le récit d'un témoin ${ }^{36}$-, devinrent trop bruyants et

\footnotetext{
${ }^{32}$ Danielle Alexandre-Bidon, «Folklore, fêtes et traditions populaires de Noël et du Premier de l'An (XIV $-\mathrm{XV}^{\mathrm{e}}$ s.) », Razo, vol. 8, 1988, p. 46.

${ }^{33}$ Graham A. Runnals, «Le mystère français : un drame romantique ? », Étude sur les Mystères, Paris, Champion, 1998, p. 26 et suiv.

${ }^{34}$ The Drama of the Medevial Church, vol. II, Oxford, Clarendon Press, 1933, p. 20 Karl Young, "Officium Pastorum: a study of the dramatic developments », Transactions of the Wisconsin Academy of sciences, arts and letters, vol. 17, part. 1, 1912, p. 299-396 ; Eric Palazzo, Liturgie et société au Moyen Âge, Paris, Aubier,
} 2000.

${ }^{35}$ Élisabeth Lalou, «Le théâtre médiéval, le tragique et le comique : réflexions sur la définition des genres », Publication numérique du CÉRÉdI, dans les actes du colloque Tragique et comique liés, dans le théâtre, de l'Antiquité à nos jours (du texte à la mise en scène), univ. de Rouen, avril 2012. Voir aussi de Janine Horowitz, «Les danses cléricales dans les églises au Moyen Age », Le Moyen Age, $\mathrm{n}^{\circ} 2,5^{\mathrm{e}}$ série, t. III, 1989, p. 279-292.

${ }^{36}$ Récit de Paul Ourliac cité par J.-P. Piniès, Languedoc méditerranéen, Paris, Bonneton, 1989, p. 159: «En mémoire des pasteurs qui vinrent visiter le Sauveur dans son étable, on entendit tout à coup résonner sous les immenses voûtes gothiques le bruit discordant d'une multitude de sifflets, signal de l'arrivée des pâtres des campagnes voisines. Puis du fond de l'église, on vit s'avancer en bon ordre et processionnellement une foule de bergers, de chevriers, de pâtres de montagne en habits de fête, avec leurs bâtons ferrés, leurs capes, leurs cornemuses, et conduisant en laisse de petits agneaux blancs comme neige, tout parés de nœud et de rubans. L'orgue 
divertissants. Ils engendraient des superstitions : par exemple, le berger qui se présentait le premier à l'offrande était assuré d'avoir les plus beaux agneaux de la paroisse. Ces désordres causèrent leur disparition progressive, entre autres par un arrêt du Parlement de Paris en 1548 et un édit en $1677^{37}$. Toutefois, au début du $\mathrm{Xx}^{\mathrm{e}}$ siècle, on remit au goût du jour les «pastrages », offrandes de l'agneau empreintes de sérieux, ainsi aux Baux-de-Provence ${ }^{38}$.

Les jeux liturgiques encouragèrent les crèches, représentations sages de la Nativité. Le mot vient de l'allemand Krippe ${ }^{39}$, mangeoire pour animaux: le premier à utiliser le mot comme «mangeoire pour l'Enfant-Jésus » fut Gautier de Coincy au début du XIII ${ }^{\mathrm{e}}$ siècle $^{40}$. On n'emploie jamais ce mot pour parler de peintures. Les «crèches », petits théâtres avec figurines mobiles qu'on dispose au moment de Noël, et emploi métonymique du mot de la partie pour le tout, se sont progressivement imposées, d'abord dans les églises à partir du XVI e siècle, comme à Chaource (Aube) vers 1540 ou à Nogent-le-Rotrou (Eure-etLoir) à la fin du siècle. Les statues pouvaient être de grandeur nature. Par la suite, selon des exemples napolitains en particulier, on prit l'habitude de la faire dans les familles avec des figurines en argile de hauteurs variables, de 10 à $35 \mathrm{~cm}$, surtout à partir du XIX ${ }^{\mathrm{e}}$ siècle.

Il y avait de nombreuses légendes de merveilles qui se produisaient à minuit : les pierres s'ouvraient sur des trésors, des arbustes fleurissaient dans la nuit, les animaux se parlaient dans les étables, et malheureux celui qui voulait surprendre leur conversation car il mourrait dans l'année, ainsi

aussitôt entama bruyamment l'air d'un joyeux noël, et tous les pâtres entonnèrent de concert. »

${ }^{37}$ Françoise Lautman, op. cit., p. 37.

38 Régis Bertrand, «Les cérémonies d'offrande à la messe de minuit 》, Siècles, vol. 21, 2005, p. 109-123.

${ }^{39}$ Voir le Trésor de la langue française informatisé.

${ }^{40}$ Gautier de Coincy, Miracles, éd. Frederic Kœnig, t. IV, 559, 322, cité par le Trésor de la langue française. 
qu'en témoigne encore George Sand en Berry en $1852^{41}$.

Pour remplacer les jeux trop bruyants, les chants de Noëls, appelés noëls (avec un $\mathrm{n}$ minuscule), apparurent à partir $\mathrm{du} X V^{\mathrm{e}}$ siècle et se multiplièrent au siècle suivant. S'y trouvaient transposées des scènes inspirées des jeux liturgiques et de leurs dialogues. Ils étaient consignés dans des recueils, les Bibles de Noël, qui se sont multipliés avec l'invention de l'imprimerie à la fin $\mathrm{du} \mathrm{XV}^{\mathrm{e}}$ siècle ${ }^{42}$. Ces noëls étaient composés en langue régionale par des poètes, curés de campagne, organistes ou simples amateurs sur des airs souvent profanes et préexistants. Rabelais fit allusion à ceux du Poitou dans l'«Ancien Prologue » du Quart Livre en 1548: «En Angiers estoit pour lors un vieux oncle, seigneur de Sainct George, nommé Frapin c'est celuy qui a faict et composé les beaux et joyeux Noelz en langage poictevin ». Ce Frapin semble être l'abbé Lucas Le Moigne, curé de Saint-Georges-duPuy-la-Garde ${ }^{43}$ à la fin du Xv ${ }^{\mathrm{e}}$ siècle, auteur de noëls originaux.

\section{Les traditions domestiques et sociales}

Selon leur étymologie, les traditions domestiques sont celles qui sont vécues à la maison, en famille. Les traditions sociales, pour leur part, concernant le village entier, mettent en valeur en particulier le rôle essentiel tenu par les enfants en cette période de l'année.

Aujourd'hui saint Thomas,

Cuis ton pain,

\footnotetext{
${ }^{41}$ Récit de George Sand, illustré par Maurice Sand, paru dans L'Illustration du 25 décembre 1852.

${ }^{42}$ Henry Poulaille les a soigneusement recueillis dans La Grande et belle bible des noëls anciens, du XII ${ }^{e}$ au XVI ${ }^{e}$ siècles, Paris, Albin Michel, 1942 ; XVII et XVIII siècles, 1950 ; Noëls régionaux et noëls contemporains, 1951 ; Amédée Gastoué, Le Cantique populaire en France. Ses sources, son histoire augmentés d'une bibliographie générale des anciens cantiques et Noëls, 1925 ; Marina Fey, Noëls en français et en dialectes du XVI siècle, Lyon, Centre d'études linguistiques Jacques Goudet, 2004. Voir les travaux de Marie-Noëlle Colette.

${ }^{43}$ Saint-Georges des Gardes, Maine-et-Loire.
} 
Lave tes draps.

Comme l'indique en partie ce dicton, on blanchissait son linge et on s'habillait de neuf pour Noël ${ }^{44}$. La période de Noël était en effet un temps nouveau, temps particulier ouvert sur l'au-delà et temps de divination où l'on disait que le ciel s'entrouvrait. Gagner aux jeux portait bonheur : on jouait beaucoup, aux dés surtout. La fête de Noël occasionnait également un grand repas réunissant la famille. Ces traditions se voulaient toutes annonciatrices de bonheur et de prospérité. Comme les Romains de l'époque impériale, le repas était prometteur. Lors de la Tabula fortunata, à la veille des Calendes de janvier et donc d'une nouvelle année, le sens augural était très présent: l'abondance promettait l'abondance ${ }^{45}$. Bien avant l'institution légale de l'année au $1^{\mathrm{er}}$ janvier $^{46}$ c'était l'avènement d'un renouveau puisque dans l'hémisphère nord les jours rallongent. Les banquets nocturnes et autres réjouissances profanes à cette époque du solstice d'hiver étaient dénoncés pour leurs superstitions par les Pères de l'Eglise, par Tertullien aux II $^{\mathrm{e}}$ et III $^{\mathrm{e}}$ siècles, par Alexandre Sévère à la fin du IV siècle, ainsi que par saint Jérôme († v. 420) et Césaire d'Arles.

Dans les traditions domestiques que nous connaissons encore aujourd'hui, celle de l'arbre coupé en nombre à l'occasion de Noël est attestée à partir de 1521 à Sélestat en Alsace ${ }^{47}$ : les comptes de la ville font état de deux schillings à payer aux forestiers pour surveiller l'abattage de «mais » (arbres) le jour de la Saint-Thomas (à l'époque, le 21 décembre). Des arbres entiers étaient déjà attestés dans des bâtiments publics : halls d'hôpitaux ou maisons de confréries. Ainsi en Allemagne, la confrérie des garçons boulangers en offrit-elle un, garni de bretzels, à

\footnotetext{
${ }^{44}$ Roger Vaultier, Le Folklore pendant la Guerre de Cent Ans, op. cit., p. 83.

${ }^{45}$ Françoise Monfrin, «La fête des calendes de janvier, entre Noël et Épiphanie », La Nativité et le temps de Noël. Antiquité et Moyen Âge, Aix-en-Provence, Publications de l'Université de Provence, 2003, p. 112-114.

${ }^{46}$ En 1564 en France sous Charles IX.

${ }^{47}$ Gérard Leser, Noël-Wihnachte en Alsace, Mulhouse, Éd. du Rhin, 1989, p. 70. 
l'hôpital du Saint Esprit de Fribourg-en-Brisgau en $1419^{48}$. La tradition de mettre dans la maison des branchages verts à cette époque de l'année, quand les arbres sont dépouillés de leur verdure, était antérieure. Déjà au II $-\mathrm{III}^{\mathrm{e}}$ siècle en Afrique du Nord (romanisée à cette époque), l'usage de mettre du laurier et des lampes dans les maisons au moment de l'Épiphanie était dénoncé par l'apologiste chrétien Tertullien qui condamnait cette coutume païenne en vigueur parmi les chrétiens de son temps ${ }^{49}$. Au IV siècle en Syrie, saint Ephrem observait la coutume d'orner les maisons de couronnes au moment du 6 janvier, mais il se réjouissait, lui, de «la plus sublime des fêtes chrétiennes » où régnait une joie immense et où les enfants n'exprimaient que des paroles d'allégresse ${ }^{50}$.

Indépendamment de la verdure, brûlait dans la cheminée la bûche de Noël, centre de la veillée dans les familles. Pouvant apporter aussi bien « danger que bénédiction ${ }^{51}$ », la busche de Noël est mentionnée en 1691 dans le dictionnaire de Furetière $^{52}$, mais elle était connue bien plus tôt étant donné la permanence de cette coutume dans toutes les provinces de France et même dans une partie de l'Europe ${ }^{53}$. La coutume était très «populaire au Moyen Âge» comme le dit une lettre de rémission du Trésor des Chartes citée par Roger Vaultier. Celui-ci ajoute « cette souche ou chouque est même signalée dans de nombreux droits seigneuriaux, dès le XIII ${ }^{\mathrm{e}}$ siècle, par exemple en Normandie ${ }^{54} \gg$. Encore en 1866, selon Amédée de Ponthieu, quelques seigneurs de fiefs des environs de Paris se

\footnotetext{
${ }^{48}$ Ibid.. p. 91.

49 Tertullien, Euvres de Tertullien, Ch. XIV et XV, t. II, «De l'idolâtrie », trad. Eugène-Antoine de Genoude. Paris/Chalon-sur-Saône, Vivès, 1852, p. 229.

${ }^{50}$ Francis Weiser, «Le folklore de l'Avent et de Noël », La Maison-Dieu, vol. 59, 1959, p. 105 ; Oscar Cullmann, La Nativité et l'arbre de Noël. Les origines historiques, Paris, Le Cerf, 1993, p. 38.

${ }^{51}$ Karin Ueltschi, Histoire véridique du Père Noël. Du traîneau à la hotte, Paris, Imago, 2012, p. 105.

52 Tome I, non paginé.

${ }^{53}$ Yvonne de Siké, Fêtes et croyances populaires en Europe, Bordas, 1994, p. 53.

${ }^{54}$ Roger Vaultier, Le Folklore pendant la Guerre de Cent Ans, op. cit., p. 86
} 
faisaient apporter une bûche par leurs sujets, la veille de Noël ${ }^{55}$.

Noël engendre de grands repas familiaux, occasions de se réchauffer, de même que la Saint-Sylvestre et, dans une moindre mesure, la fête de l'Épiphanie où l'on partage le gâteau des Rois dans une fête qui s'appelle «Le Roi boit». Le mot réveillon, «petit repas que l'on fait la nuit en compagnie $»^{56}$ au XVI $\mathrm{e}^{\mathrm{e}}$ siècle, ne prit qu'au XVIII ${ }^{\mathrm{e}}$ le sens de repas nocturne de Noël puis de la Saint-Sylvestre. La préparation du grand repas était importante comme le montrent de nombreuses miniatures où la « tuerie» du cochon et le festin figurent souvent pour illustrer décembre et janvier. Nombreuses sont les mentions de suppléments que le repas de Noël permettait. Déjà à l'époque carolingienne, l'abbé Théodemar en 797 nous apprend que, selon la Règle de saint Benoît, les moines mangeaient de la volaille à Noël et pendant l'octave qui suit, et de même à Pâques ${ }^{57}$. Le Roman de Renart aux $\mathrm{XII}^{\mathrm{e}}$ et $\mathrm{XIII}^{\mathrm{e}}$ siècles cite à la branche VII, l'« oie grasse » de Sire Gombert, paysan des bords de l'Oise près de Compiègne, «qu'il a fait engraisser sans regarder à la dépense » pour la manger à Noël, et, plus loin, branche XII : «Ce fut un pou devant Noël / Que l'on metoit bacons en $\operatorname{sel}^{58} »$. Comme pour d'autres grandes fêtes en milieu urbain, il y avait au XIII ${ }^{\mathrm{e}}$ siècle, à côté de la charcuterie, une viande noble de bœuf ou de mouton : les statuts du chapitre de la cathédrale de Nice en 1233 prévoyaient pour Noël, Pâques et la Pentecôte « deux viandes, de la mortadelle et des légumes $»^{59}$. Les moyens de les acquérir n'étaient pas

\footnotetext{
${ }^{55}$ Amédée de Ponthieu, Les Fêtes légendaires, op. cit., p. 35.

56 Charles de Bourdigné, La Légende joyeuse de maistre Pierre Faifeu, éd. Francis Valette, Genève, Droz, 1972 [1532], p. 30 (cité par le Trésor de la langue française).

${ }^{57}$ Michel Rouche, «Les repas de fête à l'époque carolingienne » dans Manger et Boire au Moyen Âge, Actes du Colloque de Nice (1982), dir. Denis Menjot, Paris, Les Belles Lettres, 1984, p. 266.

58 Vers 1174 pour les branches les plus anciennes; Roman de Renart, éd. Jean Dufournet, Paris, Flammarion, 1985, t. 2, p. 23.

${ }^{59}$ Danielle Alexandre-Bidon, «Folklore, fêtes et traditions populaires de Noël et du Premier de l'An, XIV ${ }^{\mathrm{e}}-\mathrm{XVI}{ }^{\mathrm{e}}$ s. », art. cit., p. 49.
} 
toujours légaux. Dans la région de Toulouse, la volaille était prisée au menu de Noël au XIV ${ }^{\mathrm{e}}$ siècle : ainsi, «en 1360, le frère convers Pierre Tarcentinus, chargé de la grange, dépose plainte contre deux frères de Gariès, Armand et Jacques Bernès, qui ont dérobé des poules et un $c o(\mathrm{sic})^{60} \gg$. Les oies grasses étaient réclamées pour Noël par les seigneurs du Bourbonnais à leurs métayers dans les contrats. L'oie n'a pas été de sitôt supplantée par les «poules d'Inde » (volailles d'Amérique centrale que Christophe Colomb appelait Inde, devenues simplement «dindes $»^{61}$ ) au XVI siècle, car leur chair n'était pas encore estimée par le médecin Charles Estienne dans la Maison Rustique (1570) ${ }^{62}$. Les animaux, que l'on choyait ce soir-là en pensant à ceux de la crèche, avaient droit également à une double ration de foin.

\section{Les étrennes}

Les étrennes, équivalentes à des vœux en nature et déjà attestées par Ovide au début de notre ère dans les Fastes ${ }^{63}$, étaient de la même façon annonciatrices de prospérité. Le mot vient de Strenia, déesse latine présidant à la bonne santé ${ }^{64}$. Selon la légende, des rameaux provenant d'un bois de bon augure qui lui était consacré auraient été offerts au roi sabin Tatius au moment de la naissance de Rome, au VIII ${ }^{\mathrm{e}}$ siècle avant J.-C. ${ }^{65}$ Roger Vaultier notait qu'au XIII ${ }^{\mathrm{e}}$ siècle, «les étrennes étaient

\footnotetext{
${ }^{60}$ Georges Passerat, «Dévotions et superstitions dans un village de Lomagne (Escazeaux) à la fin du Moyen Âge », Cahier de Fanjeaux, vol. 40, L'Église au village. Lieux, formes et enjeux des pratiques religieuses, 2006, p. 211-228.

${ }^{61}$ Rabelais, Quart Livre, éd. Robert Marichal, 1552, LX, p. 240.

62 «Vray que la chair en est délicate, mais fade \& de dure digestion [...]. Il y a trop plus de plaisir \& de bonté de chair au paon ", L'Agriculture et maison rustique, à Paris, chez Jacques du Puys, 1572, chap. 19, livre I, p. 34-35.

${ }^{63}$ Ovide, Les Fastes, op. cit., Livre I, vers 171-226.

${ }^{64}$ Germaine Guillaume-Coirier, «Arbre et herbe. Croyances et usages rattachés aux origines de Rome », Mélanges de l'Ecole française de Rome. Antiquité, vol. 104, n 1 , 1992 , p. 346.

${ }^{65}$ Symmaque († vers 402), Epist., éd. Jean-Pierre Callu, Paris, Les Belles Lettres,
} 
entachées d'idolâtrie », car on affirmait que « nul n'esteroit riches en l'an, s'il n'estoit hui estrinés », ce que le clergé considérait comme une sorcerie. Les grands personnages donnaient une somme d'argent à leurs domestiques. Ainsi, le registre des comptes d'Isabeau de Bavière pour 1416-1417 porte-t-il la mention : «à Jehan Petit pour lui et ses compagnons varles de chambre que la royne leur a donné le jour de l'an pour aller aux estuves ${ }^{66} \gg$ En retour les domestiques offraient des présents à leurs maîtres.

Les enfants, qui sont dépositaires de l'avenir comme on sait ${ }^{67}$, jouaient un très grand rôle au moment de Noël et des Douze Jours. Pour les tournées de porte en porte, très répandues dans toute l'Europe, ils se groupaient à plusieurs et, inconsciemment, on leur accordait le rôle de passeurs vers l'Au-delà ${ }^{68}$. Claude Lévi-Strauss a justement souligné leur fonction importante jouée au moment du solstice: «Mais qui peut personnifier les morts, dans une société de vivants, sinon tous ceux qui, d'une façon ou de l'autre, sont incomplètement incorporés au groupe $?^{69}{ }$. En échange de leurs vœux ou de leurs chants, il fallait leur donner un petit cadeau : une pomme, des noisettes ou des gâteaux. Sinon les enfants «dé-chantaient », c'est à dire qu'ils retiraient leur souhait en proférant des malédictions, ce qui prouve que ces quêtes étaient magiques et protectrices ${ }^{70}$. Du Cange, dans son Glossarium $(1678)^{71}$, cite à l'entrée

t. IV, X, 35 .

${ }^{66}$ Roger Vaultier, Le Folklore pendant la Guerre de Cent Ans, op. cit., p. 96, n. 3. Selon l'inventaire du prince, Pol de Limbourg, peintre du duc de Berry, avait fait don à celui-ci d'une salière d'agate garnie d'or et de pierres précieuses.

${ }^{67}$ Yves-Marie Bercé, Fête et révolte, des mentalités populaires du XVIe au XVIIIe siècle, Fayard, 1994 [1976], p. 22.

${ }^{68}$ François-André Isambert, Le Sens du sacré, Fête et religion populaire, Paris, Éditions de Minuit, 1982, p. 206 ; Martyne Perrot, Ethnologie de Noël, Grasset, 2000, p. 149.

${ }^{69}$ Claude Lévi-Strauss, «Le Père Noël supplicié », Les Temps modernes, vol. 77, 1952, p. 1588.

70 Voir Nicole Belmont, «Chanter et déchanter dans les chansons de quête», 
Kalendae, cette pratique «commune » dans la Rome antique la veille au soir du Jour de l'An, où les enfants quêtaient déjà des douceurs, « des bons ingrédients » (nous dirions des « friandises »), de porte en porte. Ces tournées subsistent encore aujourd'hui dans différents pays d'Europe, comme celle des Christmas Carols en Angleterre. Cette coutume des Aguilannées, du fameux «Au gui l'An neuf ! dit-on couramment, n'a rien à voir avec le gui des druides, mais provient du nom de la baguette écorcée, hague ou aguilaneu en patois normand, dont les enfants étaient munis $^{72}$. Les adolescents n'oubliaient pas cette coutume de leur enfance, mais leurs tournées, qui se produisaient en fin de soirée, n'avaient plus la même signification et étaient beaucoup moins sages. Ainsi, en 1397 dans la prévôté de Chateaulandon le soir du 5 janvier, veille des Rois, par exemple, la tournée du guillenleu ou du haguineneu finit mal : le groupe de jeunes gens termina la soirée au cabaret par une grave dispute, et il s'ensuivit la mort de l'un d'entre eux. Roger Vaultier cite d'autres exemples $\mathrm{du} \mathrm{XV}^{\mathrm{e}}$ siècle, en Anjou et en Poitou ${ }^{73}$. Le Conseiller au Parlement de Bretagne Noël du Fail, dans ses Propos rustiques (1547), décrit les joyeux quêteurs de l'Aguilaneu (Haguilleneuf, Hoguihanneu) dans sa région de Saint-Erblon au XVI siècle, munis de leurs bâtons, leurs broches ou leurs vieilles épées rouillées où ils piquaient les limas ou le lard, en cheminant au son du fifre et du tambour ${ }^{74}$. Ils faisaient par la suite un joyeux repas ou vendaient le produit de la quête au profit de la paroisse. Au XVIII ${ }^{\mathrm{e}}$ siècle, l'abbé Jean-Baptiste Thiers rapporte dans son Traité des jeux et divertissements qu'on pratiquait encore la fête de

Ethnologie française, Paroles d'outrage, vol. 3, 1992, p. 245-247.

${ }^{71}$ Carolo Dufresne, Domino Du Cange et al, Glossarium mediae et infimae latinitatis, Paris, Niort, Favre, 1883-1887 [1678], t. IV, col. 481a.

${ }^{72}$ Arnold Van Gennep, Bernadette Guichard, Le Folklore français, « Cycle des Douze Jours. De Noël aux Rois », op. cit., p. 2790 et 2793.

${ }^{73}$ Roger Vaultier, Le Folklore pendant la Guerre de Cent ans, op. cit., p. 94.

${ }^{74}$ Noël du Fail, Propos Rustiques, Paris, J. Picollec, 1987, p. 120-121. 
l'Aguilanneuf au début de l'année dans le diocèse d'Angers, malgré la défense énoncée au synode d'Angers en 1595: «il se commettoit autrefois quantité d'insolences dans les églises sous prétexte d'une queste qu'y s'y faisoit les premiers jours de l'année, par des jeunes gens de l'un \& l'autre sexe, \& que l'on appeloit L'Aguilanneuf ». Le synode dénonçait que « sous ombre de quelque peu de bien, il s'y (commettait) beaucoup de scandales ${ }^{75} »$.

Indépendamment de ces tournées, dans la nuit, les cadeaux de Noël étaient censés tomber du ciel et les donateurs étaient divers selon les régions, aboutissant plus tard à notre Père Noël ${ }^{76}$. Saint Nicolas, fêté le 6 décembre, a vraiment existé : il était évêque de Myre, en Asie Mineure aux $\mathrm{III}^{\mathrm{e}}-\mathrm{IV}^{\mathrm{e}}$ siècles, et, à la suite du transfert de ses restes à Bari dans les Pouilles par des marchands italiens en 1087 pour les substituer aux Infidèles, son culte s'est implanté en Europe occidentale. Sa vie a donné lieu à des légendes de générosité qui en ont fait le patron des marins et des marchands pour avoir sauvé des hommes de la tempête ou de la famine, celui des avocats pour avoir délivré trois prisonniers injustement condamnés, celui des fiancés pour avoir aidé trois jeunes filles que le père trop pauvre vouait à la débauche en mettant, anonymement, trois nuits de suite des bourses d'or sur le rebord de la fenêtre. Soulignons ici que le cadeau de nuit tombé du ciel, dont parle La Légende dorée ${ }^{77}$, ressemble fort à notre cadeau de Noël! Son patronage le plus célèbre en Occident est celui des enfants : il en a sauvé à plusieurs reprises des griffes du diable ou de voleurs. Le miracle de la résurrection des trois petits, qu'un méchant boucher (ou aubergiste) avait coupés en morceaux et mis au

\footnotetext{
75 Jean-Baptiste Thiers, Traité des jeux et divertissements..., Paris, Antoine Dezallier, 1686, p. 452 et suiv.

${ }^{76}$ Voir Martyne Perrot, Ethnologie de Noël. Une fête paradoxale,op. cit.; Nadine Cretin, Histoire du Père Noël, Toulouse, Le Pérégrinateur, 2010.

${ }^{77}$ Jacques de Voragine, La Légende dorée, éd. Alain Boureau, Paris, Gallimard, 2004, p. 29.
} 
saloir, est le plus célèbre en Occident ${ }^{78}$. Cette invention pourrait être due à la mauvaise interprétation d'une illustration des trois prisonniers à-demi sortis de leur tour, figurant en petit à côté de l'évêque ${ }^{79}$. Quoiqu'il en soit, elle a fait du saloir contenant les trois enfants l'attribut le plus fréquent de l'évêque dans les représentations occidentales. Saint Nicolas, qui fit preuve de clémence envers le criminel, est devenu par la suite patron des charcutiers, des tonneliers et des aubergistes.

Suivant un récit du moine Richer de Senones datant de 1254 environ, augmenté vers 1326 par une chronique de Jean de Bayon, de l'abbaye lorraine de Moyenmoutier, un chevalier lorrain, Aubert de Varangéville, rapporta une phalange du saint «de son doigt bénissant» (digiti benedicentis) à Port en Lorraine, non loin de Nancy, en $1098^{80}$. De là son culte s'est implanté dans les régions rhénanes, et un grand pèlerinage s'est instauré à Saint-Nicolas-de-Port - le toponyme est attesté en $1150^{81}$-, en particulier pour tous les anciens prisonniers des croisades $^{82}$. Ce pèlerinage a été très fréquenté entre autres par Joinville,

\footnotetext{
${ }^{78}$ Ce miracle n'apparaît pas dans les récits grecs et il n'apparaît pas non plus dans $L a$ Légende dorée. Il fut attesté à partir $\mathrm{du} \mathrm{XI}^{\mathrm{e}}$ siècle dans un texte de Godehard, évêque bavarois d'Hildesheim $(\dagger 1038)$. Le chanoine anglo-normand Wace, dans sa Vie de saint Nicolas (écrite vers 1150), parle, lui, de «trois clercs » et pour l'Italien saint Bonaventure $(\dagger 1274)$, il s'agit de «deux écoliers » allant à l'école d'Athènes étudier la philosophie. Voir de Colette Méchin, Saint Nicolas, Boulogne-Billancourt, BergerLevrault, 1978, p. 30.

${ }^{79}$ Charles Cahier, Caractéristiques des saints dans l'art populaire, Paris, Librairie Poussielgue Frères, 1867, p. 303 et suiv.; Colette Méchin, Saint Nicolas, op. cit., p 30.

${ }^{80}$ Colette Méchin, op. cit., p. 33 et suiv. ; Marie-Hélène Colin, Emmanuelle Friant, Philippe Martin, «La dévotion à saint Nicolas en Lorraine du XV $\mathrm{XV}^{\mathrm{e}}$ aIX ${ }^{\mathrm{e}}$ siècle », dans Francine Roze, Saint Nicolas et les Lorrains. Entre Histoire et Légende, Metz, Édition Seprpenoise, 2005, p. 56.

81 Versus Sanctum Nycholaum (1150). Ernest Nègre, Toponymie générale de la France, vol. III, Genève, Droz, 1998, p. 1567.

${ }^{82}$ Une chapelle de l'hôpital du monastère bénédictin de Gorze (qui possédait Port) était consacrée depuis 1065 aux saints confesseurs dont saint Nicolas. Voir Colette Méchin, Saint Nicolas, op. cit., p. 33.
} 
Jeanne d'Arc et divers princes du Royaume ${ }^{83}$. Le culte de saint Nicolas, patron des marins, s'était également répandu le long de la Manche et de la Mer du Nord, en Normandie entre autres, depuis la seconde moitié du $\mathrm{XI}^{\mathrm{e}}$ siècle, par les Normands de Robert Guiscard implantés en Italie du $\mathrm{Sud}^{84}$. La célébrité de saint Nicolas a alors donné lieu à des récits célèbres permettant d'entrevoir un monde où le merveilleux l'emporte sur la vie quotidienne, dont le Jeu de saint Nicolas écrit vers 1202 par Jean Bodel, trouvère d'Arras de la fin du XII ${ }^{\mathrm{e}}$ siècle ${ }^{85}$. Dans les régions rhénanes, le culte de saint Nicolas a peu à peu recouvert certaines croyances de la mythologie germanique. Capable comme le dieu Odin de se déplacer dans les airs ${ }^{86}$, l'évêque a ainsi endossé le rôle de donateur de cadeaux en cette période noire du solstice d'hiver. Il prit également les traits de l'inquiétant Chasseur sauvage, personnage mythique connu de toute l'Europe, qui se déplaçait dans les airs par les nuits de tempête, spécialement pendant les Douze Jours entre Noël et l'Épiphanie ${ }^{87}$. Attesté par des récits monastiques et laïcs, le Chasseur sauvage était bien présent dans les croyances au milieu du Moyen Âge, comme l'a montré JeanClaude Schmitt, et celles-ci ont parfois perduré jusqu'au XIX ${ }^{\mathrm{e}}$ siècle en Alsace $^{88}$. Le bruyant équipage du Chasseur de la nuit, à la tête d'une

${ }^{83}$ Marie-Hélène Colin, Emmanuelle Friant, Philippe Martin, «La dévotion à saint Nicolas en Lorraine du XV $\mathrm{XV}^{\mathrm{e}}$ au XIX ${ }^{\mathrm{e}}$ siècle », art. cit., 2005, p. 58-60.

${ }^{84}$ Karl Meisen, Nikolauskult und Nikolausbrauch im Abendlande, Düsseldorf, Schwann, 1981 [1931], p. 289 et suiv.

${ }^{85}$ Albert Pauphilet, Jeux et Sapience du Moyen Âge, Le jeu de saint Nicolas de Jean Bodel, Gallimard, «Bibliothèque de la Pléiade », p. 43-107.

${ }^{86} \mathrm{Ce}$ dieu à la figure complexe, maître de la mort et de la guerre, veillait sur la fécondité. Il se déplaçait d'un monde à l'autre, dans les airs et sur les eaux sur son cheval à huit jambes, Sleipnir : Régis Boyer, Héros et dieux du Nord, Flammarion, 1997, p. 116.

${ }^{87}$ Gérard Leser, Noël - Wihnachte en Alsace, op. cit., p. 122-124; Arnold Van Gennep, Bernadette Guichard, Le Folklore français, Robert Laffont, 1999 [1988], vol. 3, p. 2705-2709.

88 Jean-Claude Schmitt, Les Revenants. Les vivants et les morts dans la société médiévale, Paris, Gallimard, 1994, p. 132 et suiv. Arnold Van Gennep cite l'exemple de Duzenheim (Bas-Rhin), où la croyance était encore si vivante au début du 
inquiétante armée de revenants, se déplaçait dans un vacarme d'armes, de chaînes, de cris et d'aboiements qu'il valait mieux éviter : c'était, selon les régions, la Mesnie Hellequin, la Chasse-Arthur, ou celle du roi Hérode. L'attelage perdit par la suite tout caractère diabolique et effrayant pour donner naissance tardivement au traîneau du Père Noël. Le rapprochement avec la figure du Chasseur fantôme appartenant à l'hiver et à un temps immémorial prouve que l'on accordait au distributeur de cadeaux, proche des défunts, une dimension grave et profonde. À l'époque du solstice d'hiver, revenaient les inquiétudes liées à l'obscurité qui règne chaque jour davantage. Le besoin d'abondance était crucial en cette époque où, malgré les greniers encore bien remplis, l'on ne savait pas si la vie allait reprendre normalement au printemps et si les réserves seraient suffisantes. Saint Nicolas a christianisé le généreux personnage du Carnaval - un autre début d'année - beau et bien habillé, qui distribue ses noix, ses oranges ou ses gâteaux avant l'arrivée du printemps. Encore présentes dans les carnavals européens, les personnifications pourvoyeuses d'abondance au cœur de l'hiver, venues d'un ailleurs mal défini, jouent toutes le même rôle de rassurer avec leurs cadeaux alimentaires: les «beaux ${ }^{89}$, en nombre souvent, incarnent l'année nouvelle, féconde et prospère, et les «laids », leurs affreux accompagnateurs, symbolisent le monde inconnu et non-civilisé de l'Au-delà. Les sombres acolytes représentent, eux, la figure inquiétante de l'homme sauvage venu du monde non-civilisé, de l'ailleurs, du monde des ancêtres ${ }^{90}=$ Tout naturellement, saint Nicolas s'est ainsi doublé par la même occasion de

$\mathrm{XIX}^{\mathrm{e}}$ siècle que des tsiganes l'ont utilisée pour piller le village. (Arnold Van Gennep, Bernadette Guichard, op. cit., vol. 3, p. 2707).

${ }^{89}$ Cette distinction entre «beaux » et «laids » a été faite en particulier par J.-G. Frazer, Le Rameau d'or, «Le bouc émissaire », Paris, Laffont, 1983 [1935], p. 567 et suiv. ; et par Julio Caro Baroja, Le Carnaval, Paris, Gallimard, 1979, p. 197 et suiv.

${ }^{90}$ Impossible de dater ces personnages, malheureusement : mais si les attestations sont tardives (XVIII ${ }^{\mathrm{e}}$ siècle), nul doute qu'ils existaient auparavant. 
l'énigmatique personnage dont il n'a pu se démarquer, baptisé Père Fouettard ou de divers autres noms: Hans Trapp, Knecht Ruprecht, Schmutzli, Krampus...91.

C'est un autre trouvère d'Arras de la fin du XIII siècle, Adam de La Halle (Adam le Bossu), qui mentionne la plus ancienne personnification de Noël, Sires Noeus, dans une chanson de quête en dialecte picard :

No [notre] sires Noeus

Nous envoie à ses amis

C'est as amoureux

Et as courtois bien apris

Pour avoir des Paradis [des parisis ?]

A no herluison [au choix $]^{92}$.

Mais si la présence des personnifications de l'hiver apportait l'abondance espérée et matérialisée par des gâteaux ou des oranges, l'époque entière du solstice était inquiétante.

\section{Les « Douze Jours »}

Un décret du concile de Tours de 567 nous apprend que chaque jour était fêté entre Noël et l'Épiphanie le 6 janvier, appelée principalement «fête des Rois » au Moyen Âge. Ce cycle, appelé plus tard des «Douze Jours », a été christianisé dès le $\mathrm{V}^{\mathrm{e}}$ siècle comme le précise Louis Duchesne $^{93}$. Ces jours étaient marqués par la Trêve de Noël avec

${ }^{91}$ Catherine Lepagnol, Biographies du Père Noël, Hachette, 1979, p. 75 et 109. Quand le saint évêque venait seul avec son âne, il tenait lui-même à la main un bouquet de verges pour les enfants désobéissants, comme Hans Trapp ou Knecht Ruprecht, qui pouvaient porter dans une main la hotte ou le sac de jouets, et dans l'autre les baguettes.

92 Arnold Van Gennep, Le Folklore français, vol. 3, p. 2383 (n. 5) et p. 2412. Les parisis étaient une monnaie de cette époque. Le Père Noël ne prendra forme que plus tard, en particulier à partir de 1822 aux États-Unis avec le poème d'un pasteur américain Clement C. Moore qui écrivit pour ses enfants, A Visit from St Nicholas, et les illustrations de Thomas Nast dans dans Harper's Weekly entre le 3 janvier 1863 et 1886 ; Catherine Lepagnol, op. cit. , p. 103 et suiv.; Nadine Cretin, Histoire du Père Noël, Toulouse, Le Pérégrinateur, 2010.

${ }^{93}$ Can. 18, Concile de Tours II. Louis Duchesne, Origines du culte chrétien, Étude sur la Liturgie latine avant Charlemagne, Paris, Fontemoing, 1909, p. 271-274; Jean 
suspension de toute activité guerrière, comme l'a rappelé le concile de Charroux (Poitou) en 989. Selon la croyance populaire, le temps qu'il faisait pendant ces Douze Jours annonçait le climat des douze mois de la nouvelle année, ce qui n'est pas vérifié par la météorologie ${ }^{94}$ : janvier serait pluvieux si le 26 décembre était sombre, etc. Connue dans différents pays d'Europe, cette croyance était également observée en Alsace ${ }^{95}$, en Lorraine (les petits «mois »), en Franche-Comté, en Île-de-France (les Prophètes), en Aquitaine ${ }^{96}$, ainsi qu'en de nombreuses autres régions de France où elle persista jusqu'à la première moitié du $\mathrm{XX}^{\mathrm{e}}$ siècle.

Apparus très tôt dans les livres liturgiques, les jours suivant Noël représentaient un temps de loisir particulier avec une discipline assouplie pour les membres du clergé qui, à partir du XI siècle environ, élisaient leurs prélats festifs : «pape de Saint-Etienne », «archevêque de SaintJean », etc. ${ }^{97}$ Le lendemain de Noël, le 26 décembre, la Saint-Etienne, était la fête des diacres. Roger Vaultier citait l'exemple à Melun, pour la Saint-Etienne de 1366, d'une danse à laquelle assista un homme vêtu en femme, couvert d'un pelicon (manteau), le poil par-dessus, et le visage couvert d'une coiffe : déguisement où l'on reconnaît l'inversion chère au Carnaval, comme l'est celle des sexes, des âges, des hommes/animaux ${ }^{98}$. La Saint-Jean l'Évangéliste, le 27, était la fête des prêtres. La fête des Saints-Innocents, le 28 décembre, était celle des enfants de chœur, des pueri, clercs n'ayant pas reçu les ordres majeurs. La fête de l'Enfant-Évêque jouée dans la plupart des cathédrales le jour des

Gaudemet, Brigitte Basdevant, Les Canons des conciles mérovingiens, VI ${ }^{e}-V I I^{e}$ siècles, Paris, Le Cerf, 1989.

94 Jean-Philippe Chassany, Dictionnaire de météorologie populaire, Paris, Maisonneuve et Larose, 1968, p. 252.

${ }^{95}$ Selon le témoignage d'un médecin strasbourgeois du XVI ${ }^{\mathrm{e}}$ siècle, le docteur Sebig, cité par Arnold Van Gennep, op. cit., p. 2727.

${ }_{96}$ Arnold Van Gennep, Bernadette Guichard, op. cit., p. 2727 et suiv.

${ }^{97}$ Yves-Marie Bercé, Fête et Révolte, op. cit., «Les prélats festifs », p. 24 et suiv.

${ }^{98}$ Roger Vaultier, Le Folklore pendant la Guerre de Cent Ans, op. cit., p. 87. 
Saints-Innocents est connue grâce à l'explication de son déroulement dans les livres liturgiques, comme dans le Liber ordinarius ecclesiae paduane du XIII ${ }^{\mathrm{e}}$ siècle $^{99}$.

La fête des sous-diacres, la célèbre fête des Fous, avait lieu $1^{\text {er }}$ janvier (fête de la Circoncision), à l'Épiphanie, ou le 13 janvier, octave de l'Épiphanie ${ }^{100}$. Les sous-diacres étaient des hommes destinés à l'Église, mais leur fête était agitée comme toute fête d'étudiants. Déjà au $\mathrm{XII}^{\mathrm{e}}$ siècle, l'abbesse alsacienne Herrade de Landsberg s'était élevée dans son Hortus Deliciarum (vers 1170) contre les abus et «les plaisanteries grossières » qui profanaient les églises à ces occasions ${ }^{101}$. Les interdictions se multiplièrent ensuite, en particulier au concile de Bâle en 1435 (canon Turpem etiam). La fête des Fous, à laquelle l'Église s'est fermement opposée, a fait couler beaucoup d'encre à partir du XVII ${ }^{\mathrm{e}}$ siècle : on en a fait en particulier un vecteur du paganisme et elle finit par disparaitre ${ }^{102}$. La période d'inversion, où dans des mascarades « le petit devient grand », est dorénavant distincte de Noël. Elle appartient aux Jours Gras à la veille du Mercredi des Cendres, qui débute le Carême depuis le concile de Bénévent en $1091^{103}$.

Les Douze Jours ne sont plus d'actualité, et on parle simplement des «Fêtes » pour parler de la période qui va de Noël au 2 janvier. Si Noël n’a pas été inventé par le Moyen Âge, cette époque médiévale «bien

\footnotetext{
${ }^{99}$ A paraître de Yann Dahhaoui, L'Evêque des Innocents dans l'Europe médiévale, $X I I^{e}-X V^{e}$ siècle, Paris, PUF.

100 Nadine Cretin, Fête des Fous, Saint-Jean et Belles de mai, une histoire du calendrier, Paris, Seuil, 2008, p. 309-313.

${ }^{101}$ Gérard Leser, Noël-Wihnachte en Alsace, op. cit., p. 129.

${ }^{102}$ Yann Dahhaoui, «Païenne, parodique ou liturgique ? La fête des Fous dans le discours historiographique (XVII ${ }^{\mathrm{e}}-\mathrm{XX}^{\mathrm{e}}$ siècles) », Asdiwal, $\mathrm{n}^{\circ}$ 9, 2015, p. 149. Ce serait en particulier la Faculté de théologie parisienne qui, au milieu du $\mathrm{xv}^{\mathrm{e}}$ siècle, aurait obtenu son éradication grâce à une lettre de plaintes datée de 1445, mais cette lettre a été publiée pour la première fois en 1611.

${ }^{103}$ Robert Féry, Jours de Fêtes, op. cit., Seuil, 2008, p. 56.
} 
nommée peut-être en définitive ${ }^{104}$ a donné à la fête de nombreux aspects qui allaient colorer longtemps cette célébration et qui sont encore connus. Cette période de transition, de continuation, de remise en forme, de rupture vis-à-vis de l'antiquité comme de la période moderne, est comme le soulignait Jacques Le Goff en particulier celle d'un grand fossé culturel qui réside dans l'opposition entre le caractère équivoque de la culture folklorique et le «rationalisme» de la culture ecclésiastique ${ }^{105}$. À un certain nombre de rites païens liés au solstice d'hiver-pris tant aux Saturnales qu'aux mascarades du Nouvel An-, l'époque médiévale a ajouté le prisme d'un christianisme qui s'affermissait dans ses dévotions, créant d'autres traditions originales spectaculaires (jeux liturgiques, offrandes des bergers, crèches, noëls...). Ceux-ci s'enrichiront encore par la suite, surtout au XIX ${ }^{\mathrm{e}}$ siècle, avec les pastorales, pièces profanes mettant en scène des bergers se rendant auprès de l'Enfant-Jésus, mais où la crèche n'est plus qu'un prétexte. La célébrité de cette fête de la Nativité était alors principalement religieuse, mais tout était déjà en place. Les croyances et les rituels étaient scrupuleusement observés. Ce ne fut que bien plus tard, en particulier après la Seconde Guerre mondiale, que le fossé entre Noël chrétien et Noël païen allait se creuser, parallèlement à la sécularisation des sociétés qui voit un recul rapide des pratiques dévotionnelles, qu'elles soient admises par l’Église ou non ${ }^{106}$. Avec l'avènement des préoccupations matérielles de nos sociétés «de consommation », le sens de la fête s'est tourné vers les cadeaux et le Père

\footnotetext{
${ }^{104}$ Jacques Le Goff, Pour un autre Moyen Âge, Paris, Gallimard, 1977, p. 338.

${ }^{105}$ Ibid., p. 229.

${ }^{106}$ Par exemple, en 1952 dans un article des Temps Modernes ( ${ }^{\circ} 77$, p. 1575 et suiv.) «Le Père Noël supplicié », Claude Lévi-Strauss souligna un fait divers arrivé le 23 décembre 1951 à Dijon. Après avoir été condamné par le clergé local d'usurpateur et d'hérétique, le Père Noël en effigie a été brûlé sur le parvis de la cathédrale devant les enfants des patronages. Cette surprenante affaire rappelait les manifestations antipaïennes du Moyen Âge, comme l'écrivait Jacques Berlioz dans un article de L'Histoire de 2001, (n 260, p. 28-29).
} 
Noël, avatar de saint Nicolas, mais Noël, fête très complexe, est loin de se limiter à cet aspect commercial. 